\title{
A formalised model of the information and materials handling activities in the construction process
}

\section{Construction Innovation, Arnold Journals, Volume 2/3, September 2002, pp. 133-149}

This is a personal version (Author's manuscript after acceptance for publication but before final proofreading and typesetting) of the article

\author{
Bo-Christer Björk \\ HANKEN School of Economics \\ Helsinki, Finland \\ Bo-Christer.Bjork@hanken.fi
}

\begin{abstract}
A model of the information and material activities that comprise the overall construction process is presented, using the SADT activity modelling methodology. The basic model is further refined into a number of generic information handling activities such as creation of new information, information search and retrieval, information distribution and person-toperson communication. The viewpoint could be described as information logistics. This model is then combined with a more traditional building process model, consisting of phases such as design and construction. The resulting two-dimensional matrix can be used for positioning different types of generic IT-tools or construction specific applications. The model can thus provide a starting point for a discussion of the application of information and communication technology in construction and for measurements of the impacts of IT on the overall process and its related costs.
\end{abstract}

Keywords. Construction process, information technology, SADT, information logistics

\section{Introduction}

Over the last decade and a half a number of researchers have presented formalised models of the construction process, and process modelling has become a research activity in its own right, as noted in the names of a number of recent construction IT conferences, where the terms product and process modelling often figure [i.e. Goncalves and Steiger-Garcao 2000]. There are a number of distinct reasons for the elaboration of such models, but a primary motive has been to achieve a better understanding of what activities take place on in the overall construction process and how these could be supported by or made more efficient by the use of information and communication technology. Other important motives have included the provision of support for companies wishing to study and improve their processes.

On the international level very little has been done. The effort to note has been the draft international standard proposed by the ISO committee TC 59/SC13: Organisation of information about construction works - Part 2: Framework for classification of information [ISO 1999]. This standard is the result of work on international harmonization of building 
classifications systems, but some parts of the content has relevance also for building process modelling. The proposed standard contains a number of possible classifications of the different stages of a construction project, although there is no one clear unambiguous classification, which for instance could be used as the basis for construction process models. According to the draft standard the important classes of construction process are: management process and work process. Inside the entire life-cycle process of a building a number of stages can be observed: these include construction entity lifecycle stages and project stages.

Models on the national level include models, which have been defined by professional organisations of different sorts (often in the form of checklists) to be used as basis for definition of standard contracts etc. Alternatively there are models, which strive to model the whole construction process or constructed entity life cycle according to national practice, often with the ambition to offer a model to the industry of that country of how to organise its work. Such models have often been defined by research institutes in co-operation with industrial experts. In the following a number of such models are briefly reviewed.

- The Dutch BIM model 1987-89

- The IBPM of Penn State University

- A model of the Finnish Construction Process by VTT

- The UK Process Protocol model

The Dutch Bouw informatie model was defined in an ambitious project led by an organisation called Stichting Bouwresearch from Rotterdam during the years 1987-89. The only documentation about the project consists of two thick folders in Dutch, which makes it a bit difficult to study and evaluate the results [Merendonk and Dissel 1989]. The project developed both product and process models, using the IDEF0 and IDEF1X methodologies. There is no information available concerning the possible later use of the model. The overall focus of the model seems to be with the information flows during the design phase of the project (BIM is in essence a design process model). This is understandable since the purpose of the model was mainly to serve as a framework for how the information described in the product model part of BIM is created and managed.

Of the above models the IBPM from Pennsylvania State University has perhaps been the most influential since it has influenced a lot of later work, in particular in its use of a formalised modelling methodology [Sanvido et al 1990]. The modelling work was done in close collaboration with real projects, and the chosen approach was quite pragmatic.

The model of the Finnish Construction Process developed at the Technical Research Centre of Finland combined a number of different modelling methods, one of which was IDEF0 [Karhu and Lahdenperä 1999]. The model followed existing practice quite closely, since a major input consisted of checklists of tasks produced by the Building Information Institute, which are a sort of de-facto standard in Finland.

The UK Process Protocol has been developed as collaboration between a number of research institutes and industry [Kaglioglou et al 2000]. It differs clearly from the above approaches in it use of modelling methodology, which is based on a two dimensional table of activities. The process protocol is a normative approach, since it tries to inspire companies to use a more disciplined approach to project management (for instance a so-called "stage gate" approach), than current practice. 
In most of the above models the distinction between information processing and material processing is quite unclear. The modelling tends more to follow existing organisational borders and current documentation practise than proceed from a more fundamental analysis. In such models the design phases (which are purely information handling activities) are clearly distinguished, but it is difficult to separate out the information handling activities involved in site construction and the procurement of materials.

In this paper a slightly different approach will be tried, with the particular purpose of developing a model which could act as a starting point for a discussion of the application of information and communication technology in construction and for a measurements of the impacts of IT on the overall process and its related costs

\section{A model distinguishing information and materials processing}

In order to our modelling we first need a clear understanding of what we mean by "information technology" and "construction" and of the relationship between these two. It seems appropriate to start with construction since this is the fundamental activity to which IT techniques are applied. The purpose of construction activities is to produce artefacts such as buildings, process plants, roads and bridges. Civil engineering artefacts are, in contrast to most other manufactured products, located in particular places and need to be constructed onsite rather than in factories. They are also usually one-of a kind products. The duration of a construction project is usually long. A comprehensive definition of the construction process should clearly include the whole life cycle of civil engineering artefacts, including both design, construction, operation and maintenance. In particular it is important to stress the inclusion of operation and maintenance since an important part of the information used during these stages originates during design and construction. It is also important to include the manufacturing of the building materials needed as well as public planning and inspection activities, activities that often are overlooked in process models of construction.

Information technology (IT) can be defined as the use of electronic machines and programs for the processing, storage, transfer and presentation of information. In earlier days when the emphasis was on processing only the term electronic data processing, EDP, was common. Nowadays the use of information technology is no longer confined to huge number-crunching machines housed in air-conditioned computer halls but permeates all aspects of everyday life. Communications technology is today an important part of IT. Not only computers and their software, but also devices such as the telephone, the photocopying machine and the telefax should thus be included in the definition of information technology. Many of the functions of these devices are in fact increasingly integrated in today's increasingly sophisticated equipment, where the borders between computers, mobile phones etc are less and less clear. 
In the following the SADT modelling technique will be used as a tool for modelling the construction process. SADT is not the ideal modelling tool for this purpose, but despite some deficiencies and limitations it is easy to understand and there are good computer-based modelling tools available [Marca and McGowan 1987]. The basic methodology in SADT (or the closely related IDEF0 technique [ICAM 1981]) is to model a process as a hierarchical set of interrelated activities. In figure 1 the top level of such a hierarchical model, describing the construction process, is shown.

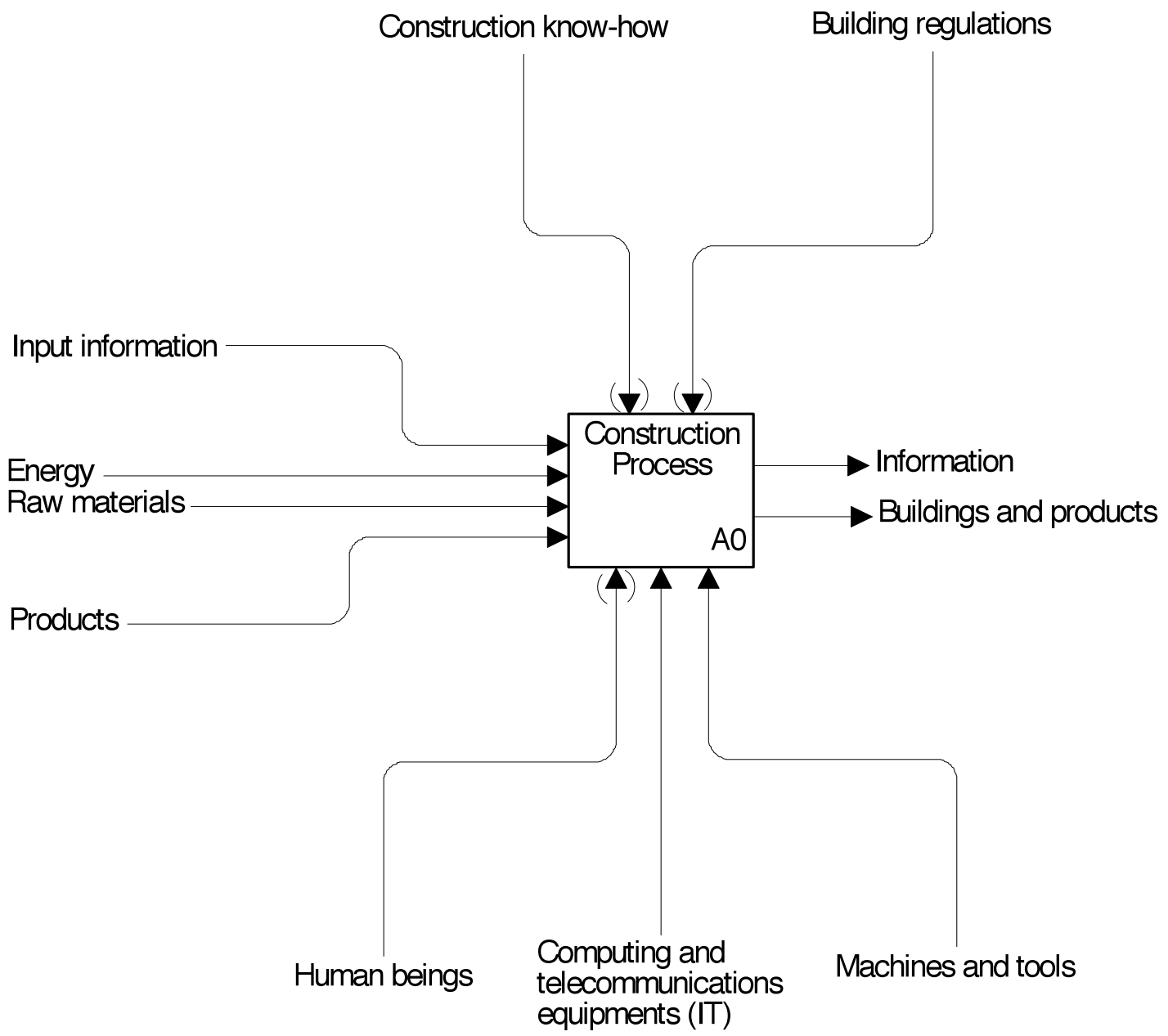

Figure 1. The construction process shows as one aggregated activity

Each activity takes some inputs (in this case input information, raw materials, etc.) and transforms these into outputs (information, buildings and products). An activity is performed by actors with the help of machines, computer software etc. These latter are called mechanisms and are shown as arrows underneath the activity box. An activity is on a more abstract level controlled by instructions or more general knowledge (controls, shown above).

In a highly abstract way the construction process can be divided into two highly integrated sub-processes, which interact with each other at many different levels. This subdivision is based on the nature of the objects that these sub-processes deal with. The information subprocess activities always result in information whereas the material sub-process activities produce services of physical objects (figure 2). 


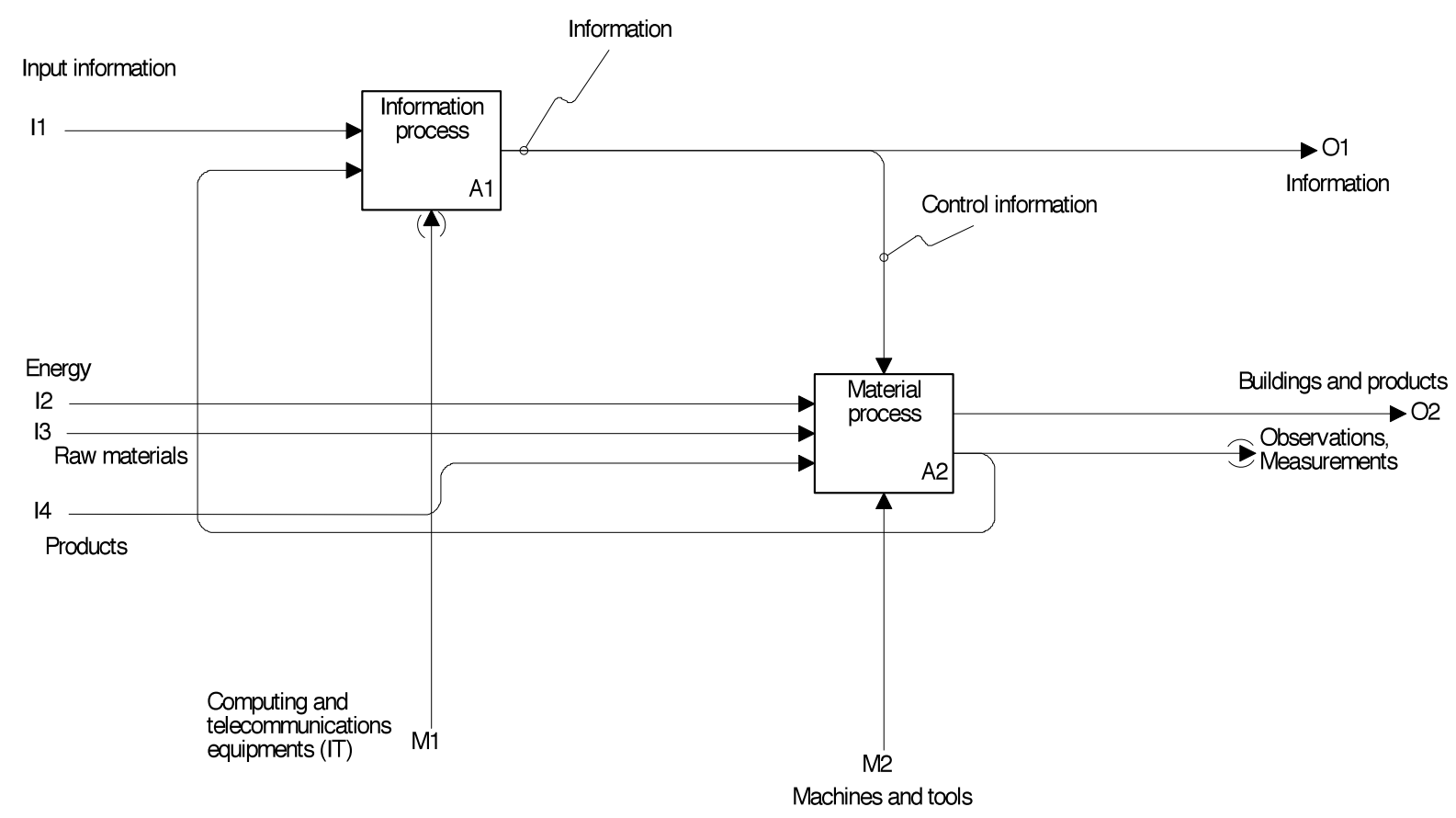

Figure 2. The construction process seen as two interacting subprocesses

In the material process raw materials and prefabricated components are created, modified, moved and installed and finally become embedded parts of the finished artefact. If we were to film a construction site during its whole duration, and to show it in extreme "fast-motion", what we would observe is almost exclusively the material process.

But the material process cannot function on its own. In contrast to the physical or chemical processes occurring in nature, the creation of any man-made artefact requires an information process, which initiates and controls the necessary material activities. The immediate results of the information process are presented as drawings, specifications, schedules, procurement orders etc. which control all material activities either by specifying the resulting artefact (design information) or the activities that need to be carried out in order for the artefact to be constructed (management information).

Both types of activities utilise resources, which are consumed in the process (materials, energy, labour, wear and depreciation of machinery). The cost of an activity is the direct result of the consumption of resources. A special type of resource or input is information, which as such isn't consumed in the process of using it, but nevertheless has a price.

The information and material sub-processes are integrated by information flows in two directions. Firstly the information process produces information, which indirectly or directly controls the material activities taking place. Secondly the information processing activities constantly need feedback information about what's actually happening in the material process, in order to check compliance with the designs or monitor the progress of the work against the schedules. In a longer time perspective the information process also needs feedback on the performance of buildings during the maintenance stages.

The interface between control information and actions in the physical world are consequently of interest. On one hand, information needs to be transformed into actions carried out by persons or by persons aided by tools and machines. The extreme case is the use of robotics, where information on higher levels needs to be transformed into very detailed instruction for how the robot moves its arms. Going in the other direction physical impulses such as temperatures, pressures, light, etc. need to be transformed into information using 
measurement equipment. The simplest transformation is done by the human eye and brain by visual observation. This mechanism can today in many cases be substituted by IT-enabled techniques such as bar code readers and automatic pattern recognition. An SADT-diagram including the interface activities is shown in figure 3.

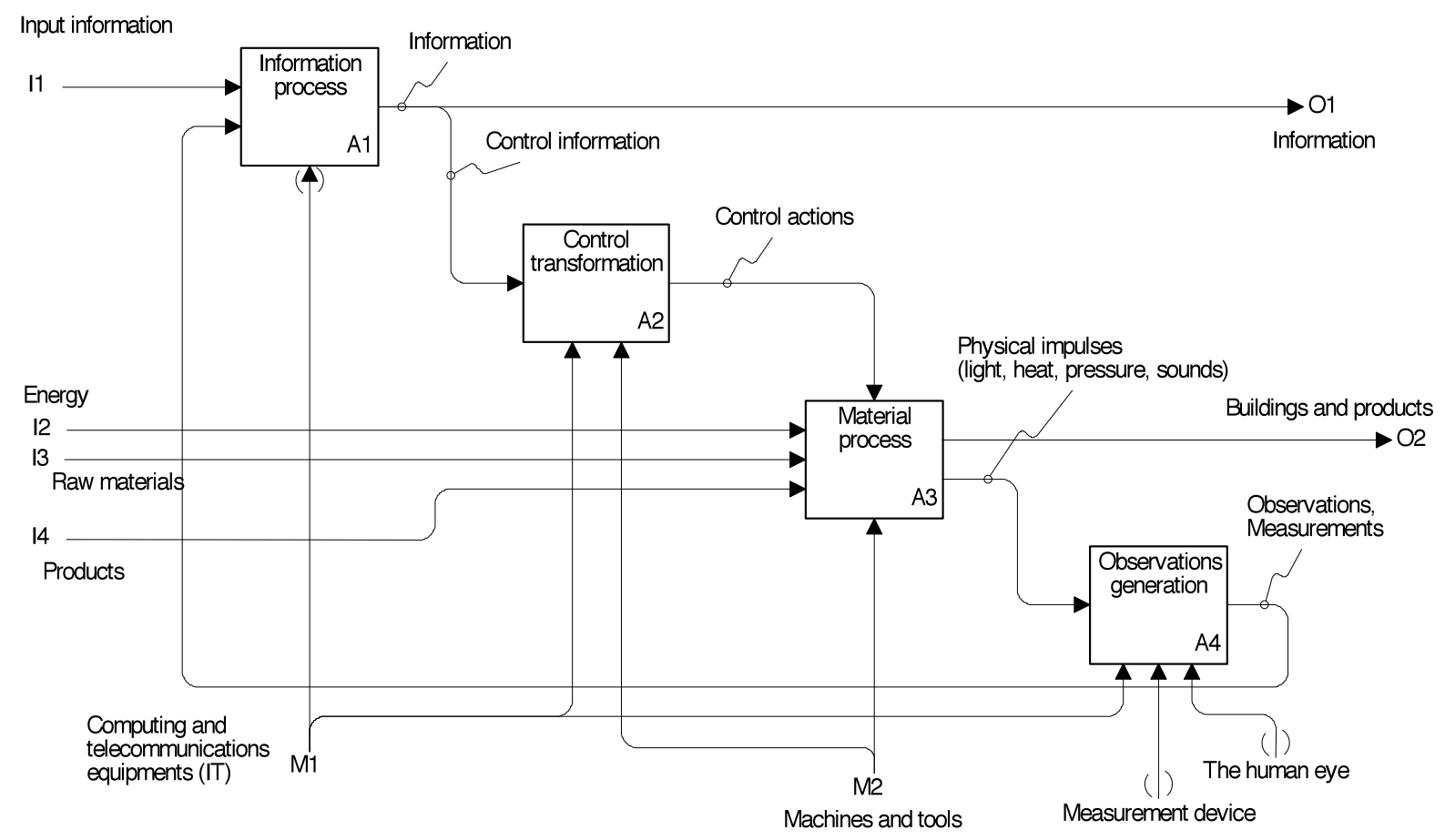

Figure 3. Interfaces between the information and material processes.

\section{Levels of abstraction in the model}

This clear split into information and material process activities can be observed on several levels of abstraction. On the highest level in our schematic model we can envisage the whole construction process, say from inception to the delivery and use of the finished artefact, which consists of a higher part aggregating the set of all information processing activities and a lower part containing all material handling activities.

If we look at the process slightly more in detail we will notice that the information process includes several consecutive and parallel activities even before any material activities start. Stages such as briefing, schematic design, tendering, etc. are basically only information processing activities and it is only in the later stages of the construction process that there is a close correspondence between information and material activities, in the sense that the information process results in detailed instructions which control material activities. The end results from an earlier information processing activity are used as input information to the next activity (for instance the client's brief as input to the schematic design stage and the architects designs as the basis for structural and building services design). In addition to product and process definition activities analysis activities which aim at predicting aspects of the material process during construction or operation of the facility (i.e. cost estimation, energy simulation, FEM-analysis) are typical in these early stages 
In later life-cycle stages we find that the information activities start to result in information, which more directly is used to control the material process, such as the procurement of materials and the construction activities on site.

At some point of detailing in our hierarchical model (i.e. weekly or daily planning of on-site activities) a stage is reached where the formalised, often company-specific documentation routines end and where oral communication starts. Much of the detailed information processing is left to the individual workers actually carrying out the tasks. This doesn't mean that the information process ends, the basic abstraction is still valid, but that at this level of detailing formal documents are no longer produced. A human bricklayer for instance doesn't require as detailed instructions as a robot doing the same job would.

An important trend of the last hundred years or so is, nevertheless, that more and more of the information needed is explicitly formulated in project documents, master specifications, etc. In earlier centuries much of the information was conveyed orally and there was heavy reliance on craftsmanship. Part of this increased degree of formalisation could also be explained by the more and complicated building services systems which need to be documented, but for the most part the underlying reason seems to be the increased division of labour in the construction industry.

In a historical perspective the construction industry shows an increasing trend to use machines to automate both the material and the information processing activities. Since the industrial revolution started in the 18th century machines have been used to automate or to aid man in performing material handling tasks. Tremendous increases in productivity have been achieved in particular in the large scale movement of materials typical of infrastructure projects.

Since the latter half of the 20th century machines have increasingly been used also to aid in information processing tasks. Early uses were in particular computer applications for engineering analysis. Since the 80's IT use, in the form of CAD and word processing software, copying machines, faxes, mobile phones, computer networks, the Internet etc. has increased enormously and now affects all aspects of the information process.

IT has in the initial stages of its introduction in the construction industry mostly been used for straightforward automation (i.e. CAD draughting). Only after a number of years have enterprises learnt about the opportunities offered by IT and started to use IT in more innovative ways. The recent developments in networking and communications technology and the miniaturisation of the hardware have also started to offer increasingly possibilities for re-engineering.

Some of the most important effects of IT on the business processes in the industry have, on the other hand, happened more or less in an unplanned fashion and not through conscious reengineering or preceded by extensive research. Consider for instance the rapid proliferation of the telefax or later Internet connections.

\section{Forms of integration}

The fundamental challenge for computer-integrated construction research is to develop techniques facilitating the efficient creation and management of information throughout the construction process [Betts and Brandon 1995].

Webster's unabridged dictionary defines integration as "to make whole or complete by adding or bringing together parts" [Webster's 1979]. Basically the goal of all conscious integration activities is to make the whole construction process function more efficiently as a whole, by 
making different sub-activities work more efficiently together as a more comprehensive system. Concurrent engineering for instance, emphasises the integration of the activities of different designers over physical distances as well as in time, using techniques such as videoconferencing, work-flow systems etc.

Forms of integration have of course been discussed by numerous authors who have presented strategies for the development of the construction process. Based on the framework presented earlier three different types of integration come to mind: integration of material process activities, integration of information process activities and organisational integration. Organisation adds a third dimension to our model, but this has not been explicitly modelled in the SADT models.

Integration of material process activities means that activities which earlier have been carried out in different places or strictly sequentially are brought together or performed in a much tighter time scale due to better co-ordination. Typical objectives of such integration is the minimisation of transportation cost of materials, decreasing the waiting times between operations, better quality control, less inventories, etc. A good example of the integration of material activities is a shift from production of building elements on site to prefabrication in factories, offering the benefits of mass production and better environmental conditions.

Integration of information activities basically means making the reuse and exchange of information between the numerous nodes that create and use information in the construction process more efficient. Using EDIFACT messages for paperless procurement of building materials, or video-conferencing for communications between designers provide good examples. Here the main objective is minimisation of the time and resources spent on information retrieval and communication.

Organisational integration can for instance be achieved by novel forms of contracting (i.e. design-build, BOT-projects) where parts of the overall process which earlier have been carried out by separate organisations are carried out within one organisation. The aim of organisational integration can for instance be to decrease transaction costs between organisations or to provide incentives for better life-cycle costing, taking into account also constructibility and maintainability during design.

These three generic types of integration usually occur in combination, not alone. In the following we shall nevertheless concentrate on the integration of information activities, since this is where IT is the primary enabling technology. It is important to remember that any larger reengineering activities aiming at integrating the information process should take account of the effects on the material process and on the organisation of the overall process.

\section{Generic information process activities}

In order to understand what integration of information activities is about and what role different sorts of IT technologies can play in such integration we need to develop further the simple model presented earlier.

In our theoretical model all activities of the information process which directly produce new information or change old information can be considered primary activities. Sometimes such activities can be carried out in relative isolation by individuals, using only their skill and knowledge as well as the computational tools directly at hand. The creative work of many major architects may belong to this category. In most cases there is, however, need for some degree of consultation with other persons or the use of input or background information, which has been created and stored earlier. Thus the primary activities, which produce new information, are almost as a rule supported by secondary activities such as communicating 
with other persons or retrieving background information. In our model we can consequently distinguish the following types of generic activities:

- Creation of new information

- Person-to-person communication

- Information search and retrieval

- Information distribution

The choice of these four categories is to some degree a matter of choice and could be criticised. The main reason for these particular subtypes is that it is relatively easy to group the application domains of general IT techniques using this classification. Thus a word processor is mainly used for the creation of new information, data base systems are vehicles of information search and retrieval, computer networks facilitate the distribution and retrieval of information and mobile phones aid in person-to-person communication.

The split into these four types of activities can be seen clearly only as we study the information process in its details. At a higher level of abstraction we find aggregated activities (for instance a task such as detailed architectural design) which in themselves consist of huge numbers of individual tasks belonging to the four categories above.

The design of the overall layout of a building is a typical aggregated activity, consisting of a large number of sub-activities from all the above categories. In addition to the actual primary decision-making activity resulting in the layout design supporting activities such as the retrieval of the city plans needed as a basis for the decision-making are needed. Communication in the form of meetings between the different designers, sending faxes to the client, etc. is also often needed in order to achieve this task. At the end of the process the resulting design solutions have to be distributed to other parties using plotters, copying services or the WWW.

An analogy from the material process would be the casting of concrete, which can be considered a primary activity since its result will directly be incorporated in the final product. The transportation of the needed materials to the site is a necessary support activity comparable to the retrieval of information.

In figure 4 these four activity types and their interrelationships are shown. Note that different types of IT-tools can be used as mechanisms for the different activities. 


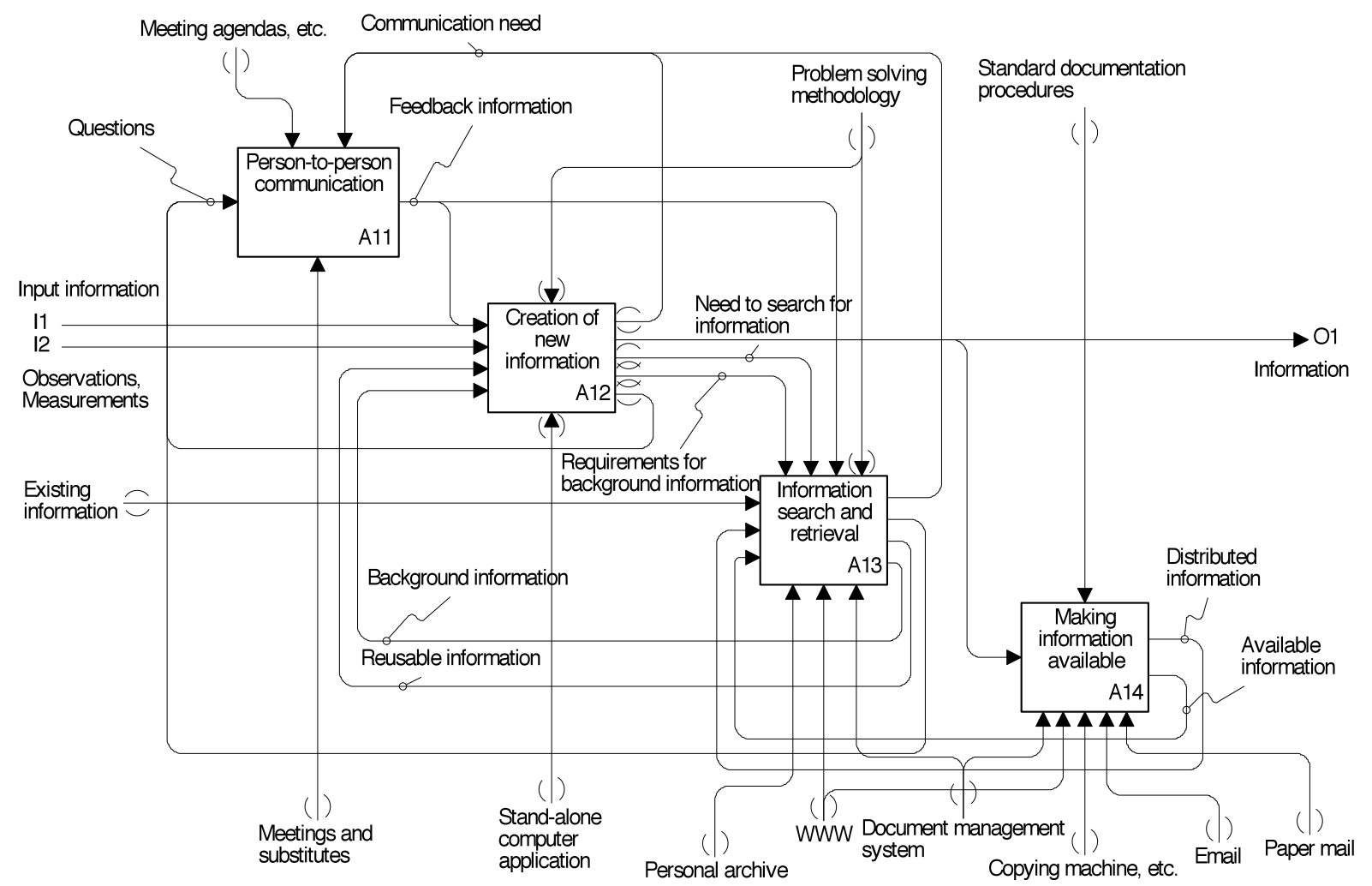

Figure 4. Four generic information process activities

Typically the creation of new information (changing old information could be seen as a special case) is carried out by one person alone either manually or with the support of a locally available computer application. To take an example close by I use a word processor for writing this presentation. Typical tools which can be of a lot of assistance to construction professionals include word-processors, CAD-systems and spreadsheets, especially when they are used to produce the first versions of documents. In later stages also the use of these kinds of applications can involve a lot of integration activities (for instance reference file techniques may be used for including data residing on remote servers into a CAD-model).

The second type of task consists of bilateral and multilateral person-to-person communication. Here the emphasis is on how IT tools can be used to support the transfer of information, rather than the creation of new information. It is important to stress that due to the fact that we have persons communicating on both sides, the information being communicated doesn't usually need to be in a highly structured form. From the viewpoint of the communication hard- and software it is enough to now that voice, raster images, video, plain text is being transmitted. It is up to the receiving person to interpret the information and to react on it.

Most modes of communication take place in simultaneous real time (a meeting, a telephone conversation, a video-conference), but we could also regard some non-simultaneous activities as essentially belonging to the same category. This is the case for modes of communication, which are cheaper and more efficient direct substitutes for simultaneous communication. These include sending and receiving letters, leaving messages in answering services, telefaxes messages and electronic mail. 
Viewed from a cost perspective communication can be seen as consisting of two generic steps; establish the communication and communicate. This is shown in figure 5. In the case of a physical meeting, for instance a meeting in the Amsterdam airport to plan a submission to a EU call for proposals, the time and cost of getting to the meeting is a substantial cost item, which could be classified under the heading establish communication. IT can very effectively be used to reduce the cost of both establishing and carrying out the communication. One of the charms of INTERNET is that it makes the effort of establishing the communication very small, compared for instance to setting up a dedicated network or modem-to-modem solutions. Video-conferencing can in the future become a quite useful substitute for physical meetings.

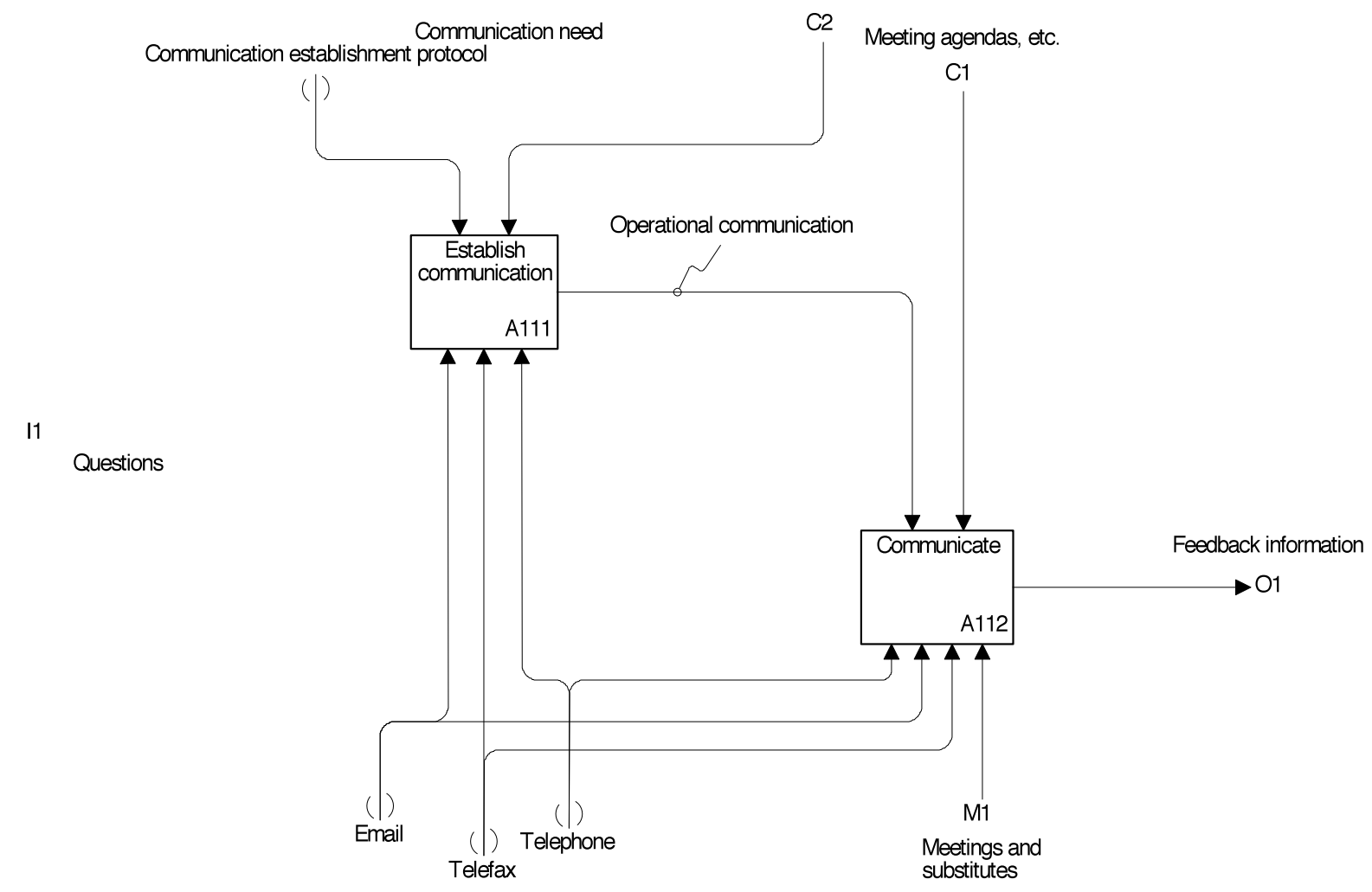

Figure 5. The subtasks of person-to-person communication.

The third type of activities consists of information search and retrieval. In order to perform some primary information creation tasks one very often needs access to information which has been created earlier and has been stored on some medium in a reusable form. In information retrieval the client can be either a person or a computer application but the provider of the information is usually some passive medium (a filing cabinet for paper documents, a drawing archive, a CD-ROM, a document management system, the World-wide web). In contrast to communication the software taking part in the activity has to know quite a lot about the information it exchanges and about its internal structure.

Let us look a little more in detail at what kind of information is retrieved. If we use the "ownership" of the information as a criterion, we could distinguish four major types: private information, project specific information, company-specific information and generally available information. Regardless of which type is involved there are a number of generic activities which have to be performed: 
- Identify relevant information

- Identify the location of the relevant information

- Retrieve the information

- Reformat the information for use

In the task identify relevant information one tries to determine which background information is of such relevance to the task at hand that it might be worthwhile to get hold of a copy. For instance if the task is to check that a particular design fulfils the building regulations this task may consist of finding out exactly which building regulations apply. The result of this task usually comes in the form of reference information. Traditionally this task could have been accomplished by asking a senior colleague, consulting the index cards of a library, etc. Today a convenient way for Web information is to use a search engine such as Lycos of Alta Vista ("building regulation+hospital").

The result of the activity identify the location of the relevant information is information as to where a copy of the information may be retrieved. In the Internet era an URL adress may be the result of this activity.

In the task retrieve the information a copy of the information is fetched so that it becomes available at the actual place where the information processing takes place. This could be achieved by having a library service mail a book or by asking a courier to deliver a copy of a drawing. Computer networks are very cost-effective in this sort of tasks, for instance using Ftp transfer.

Once the information is finally available locally there may be a need to change its format in order to make it easier to use it as input in some information processing task (in particular if some IT application is used). There could for instance be a need to copy clauses from the building regulations to insert into a specification document, and this may necessitate getting a copy of the regulation in a digital format. A very valid example of this type of transformation is where design information is available in the form of paper drawings and needs to be digitised into a CAD-format for facilities management purposes.

The overall process of information search and retrieval is shown in figure 6 . 


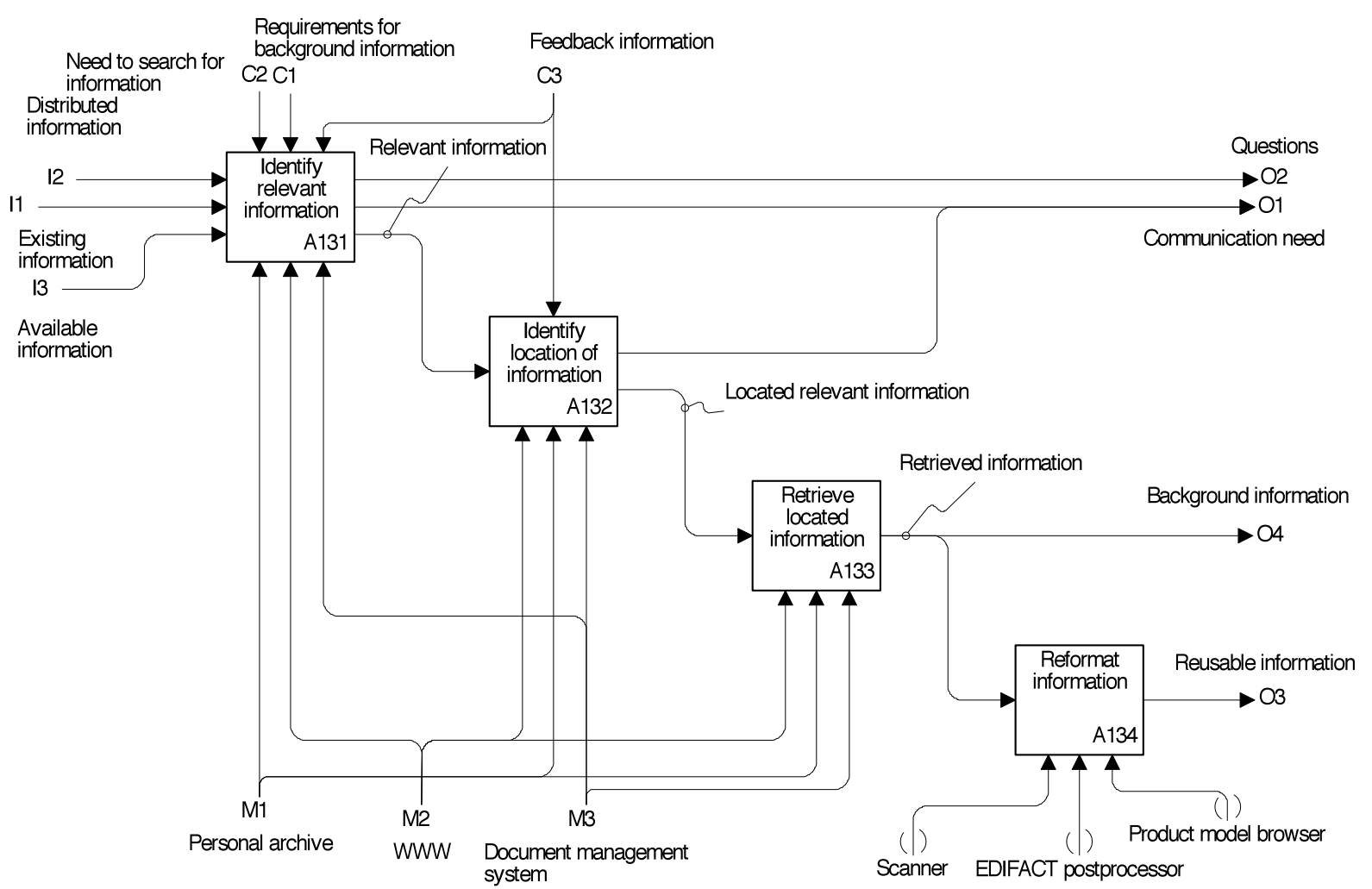

Figure 6. The activities involved in information search and retrieval.

A necessary prerequisite for information retrieval is that the needed information has already been made available by somebody else on some storage medium. This means that we also need to model the chain of activities that the producer performs after he has created some information locally. Sometimes there may also be intermediate transmitters of the information (for instance construction information services) involved in the process.

Two special cases can be discussed:

- The producer sends copies of the information directly to specific end users.

- The information is made available somewhere, but it is up to the potential end users to retrieve it.

These two special cases have been characterised as just-in-case and just-in-time distribution [Turk 1997]. In the first case the producer disseminates the information actively. The producer knows exactly who will use the information, and sends the information specifically to the receivers, using for instance internal mail in a firm, traditional paper mail, telefax or increasingly email. The responsibility for archiving the information is primarily with the end user. In many cases the information is considered unimportant and thrown in the waste paper basket, in other cases it directly calls for some action. Yet another case is when the information is filed in view of some later reuse. This mode is typically used for documents which have been produced in a project and are used as the basis of the work of other project participants, for instance drawings. The mailing of minutes from meetings is a typical example.

Just-in-case distribution involves the following types of activities.

- Format the information in a form suitable for reproduction 
- Define the reference information which identifies it

- Copy the information

- Distribute the copies

It should be noted that in this case the information retrieval activities in the receiving end are simplified since the receiver only has to search his own paper archive or local server for the information, often at some later date.

In the case of just-in-time distribution the producer doesn't know exactly who will use the information and when. This is typically the case for general construction information. In this case it is extremely important how the information producer stores the information, for instance what kind of reference information he provides the information with. Libraries, document management systems and the World Wide Web are good examples of mechanisms used for this type of information distribution.

In just-in-time distribution the producer of the information needs to perform the following tasks:

- Format the information in a form suitable for storage

- Define the reference information which helps later retrieval

- Store the information and the reference information.

The SADT diagram covering both these options is shown in figure 7.

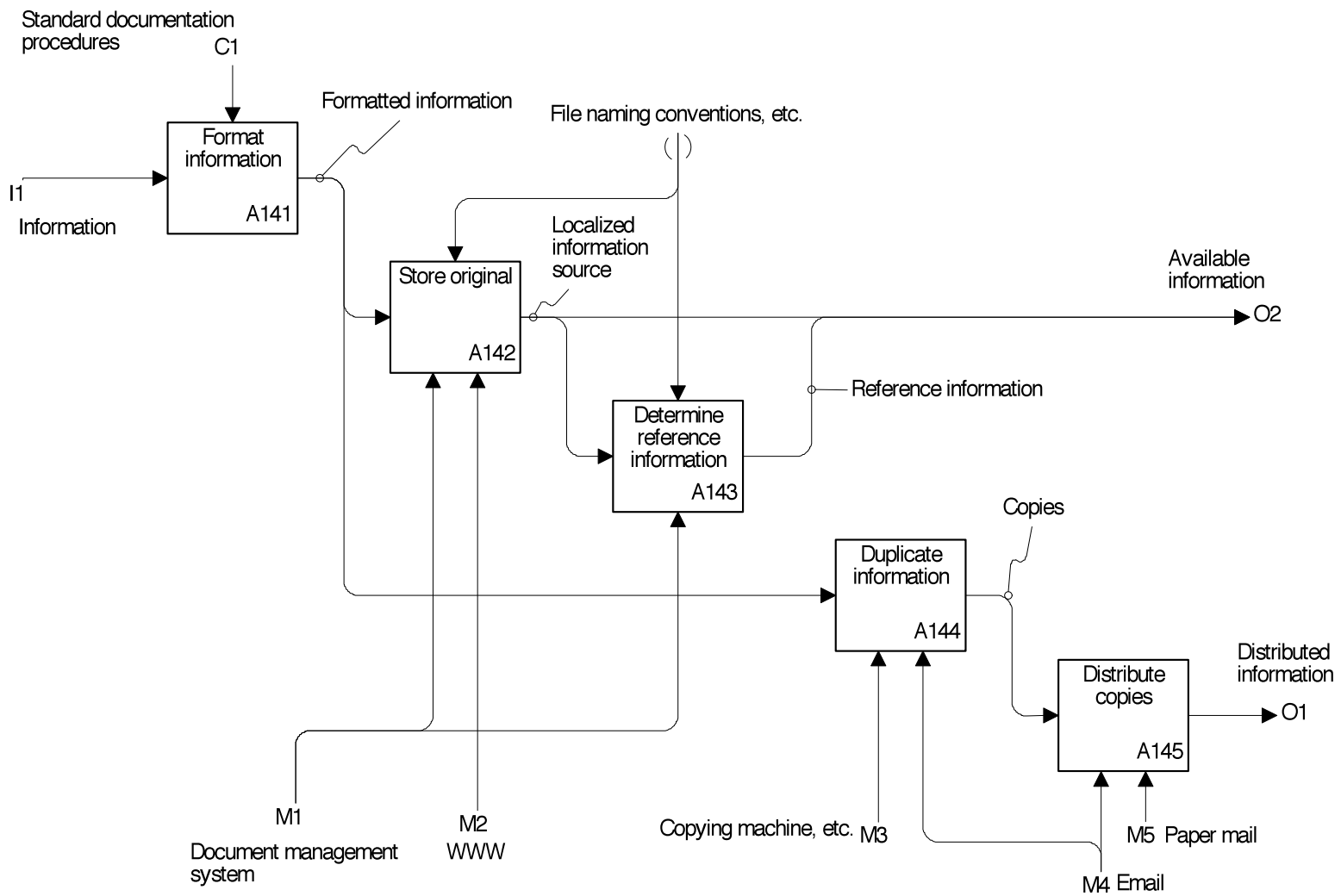

Figure 7. The activities involved in information distribution.

\section{A combined model of information logistics and process phases}


Above a rather abstract model of information management has been presented. It defines generic information management tasks from a viewpoint which could be called "information logistics".

Both communication, information retrieval and information distribution are by their very nature integration activities and can today be well supported by IT-tools. They involve the transfer of information over either physical distances or time intervals. Communication emphasises spatial integration (basically creating a situation which resembles a traditional meeting) whereas information retrieval and distribution put more emphasis on integration over distance in time. Storage technology and formalised methods of retrieval are central aspects to the latter two activities.

The information management (and also material handling) activities of the building process are traditionally modelled in a different perspective from what has been presented above, following the steps of the building process as they are generally perceived by construction professionals. Typical activities include inception, briefing, schematic design, procurement, construction, delivery, use, maintenance, demolition. Often such models aggregate information processing and material activities or creation of new information activities and retrieval activities in a way which makes it impossible to clearly distinguish between the kinds of generic information activities, which the information logistics viewpoint emphasises.

We get an interesting model if we combine our information logistics model with a traditional model of the construction process phases in an orthogonal way. This means that any activity in the construction process model (i.e. the detailed design of the structural members of a building) can in principle contain any tasks from the "Information logistics" model. We could visualise the resulting model as a matrix where the rows correspond to the categories from the information logistics model and the columns correspond to the phases of the construction process. This is illustrated by figure 8 below.

\begin{tabular}{|c|c|c|c|c|c|c|c|}
\hline \multirow{2}{*}{\multicolumn{2}{|c|}{$\begin{array}{l}\text { Generic } \\
\text { activities }\end{array}$}} & \multicolumn{6}{|c|}{ Construction life-cycle stages } \\
\hline & & Briefing & $\begin{array}{l}\text { Schematic } \\
\text { design }\end{array}$ & $\begin{array}{l}\text { Detailed } \\
\text { design }\end{array}$ & $\begin{array}{l}\text { Construc- } \\
\text { tion }\end{array}$ & $\begin{array}{l}\text { Operation } \\
\text { and use }\end{array}$ & $\begin{array}{l}\text { Decommis- } \\
\text { sioning }\end{array}$ \\
\hline \multirow{4}{*}{$\begin{array}{l}\text { Information } \\
\text { processs } \\
\text { activities }\end{array}$} & $\begin{array}{l}\text { Communi- } \\
\text { cation }\end{array}$ & & & & & & \\
\hline & $\begin{array}{l}\text { Creation of } \\
\text { new } \\
\text { information }\end{array}$ & & & & & & \\
\hline & $\begin{array}{l}\text { Information } \\
\text { search and } \\
\text { retrieval }\end{array}$ & & & & & & \\
\hline & $\begin{array}{l}\text { Information } \\
\text { distribution }\end{array}$ & & & & & & \\
\hline \multirow{3}{*}{$\begin{array}{l}\text { Material } \\
\text { process } \\
\text { activities }\end{array}$} & $\begin{array}{l}\text { Factory } \\
\text { production }\end{array}$ & & & & & & \\
\hline & $\begin{array}{l}\text { transport- } \\
\text { ation and } \\
\text { storage }\end{array}$ & & & & & & \\
\hline & $\begin{array}{l}\text { Site } \\
\text { production }\end{array}$ & & & & & & \\
\hline
\end{tabular}


Figure 8. A combined model of information logistics and process phases

Ideally this matrix should be rather large, including around 20 generic activities on the one hand and a much more detailed breakdown of the construction process on the other.Note that this model is quite general and independent of the use of IT-tools. The model thus applies equally well to the situation in 1960 or in the year 2000. Using this model we can nevertheless illustrate the importance of different types of IT-support for making the overall process more efficient. Generic IT technologies such as word processing or data bases support information logistics activities regardless of construction process phase (rows). Specific IT construction applications can be pinpointed more precisely to particular columns or even selected cells in the matrix.

\section{Conclusions}

A framework such as this could be very useful for discussing general issues related to information technology applications in construction. The model provides for instance a possible framework for measuring the effects of IT investments. In order for this to be possible, methods for measuring the activities (or resource use) in the framework would need to be developed.

The total effects of the use of IT in the construction process consists of primary effects and secondary effects. Primary effects are effects where the use of IT makes information activities (creation, retrieval, delivery and communication) more efficient. Increased productivity in the drafting work due to CAD-systems is a good example. Secondary effects are where the use of IT in information processing activities makes the material handling activities more efficient, for instance by reducing inventories, decreasing the amount of rebuilding due to contradictory design information, providing buildings that consume less energy, etc.

Using the framework we could imagine a cost breakdown of an average construction project. Such a breakdown could for instance specify that $1,3 \%$ of the overall cost is attributable for information search and retrieval activities during the schematic design stage or the $15,4 \%$ of the costs are related to the transportation and intermediate storage of building materials during construction. These figures are of course purely hypothetical. A very bold assumption could be that in the order of $50 \%$ of the total costs of the construction process are due to information activities.

The model also provides a framework for positioning diverse research topics quite precisely. For instance the role of product models, document management systems and general information services on the web can be identified.

Some directions for further work could involve:

- Developing a more detailed classification of the create information activity. This could include a division into synthesis (i.e. creating a new solution) and analysis activities (analysing the consequences of a solution), and a division into design (plan the end product) and planning (plan the activities of the process).

- Developing further a model of the material activities. Examples could be prefabrication in factories, storing material, transporting material, on-site construction work. The factor that triggers such a classification is the types of machinery and skills needed for each category a tentative diagram is shown in figure 9 (these categories were also included in the matrix in figure 8). 


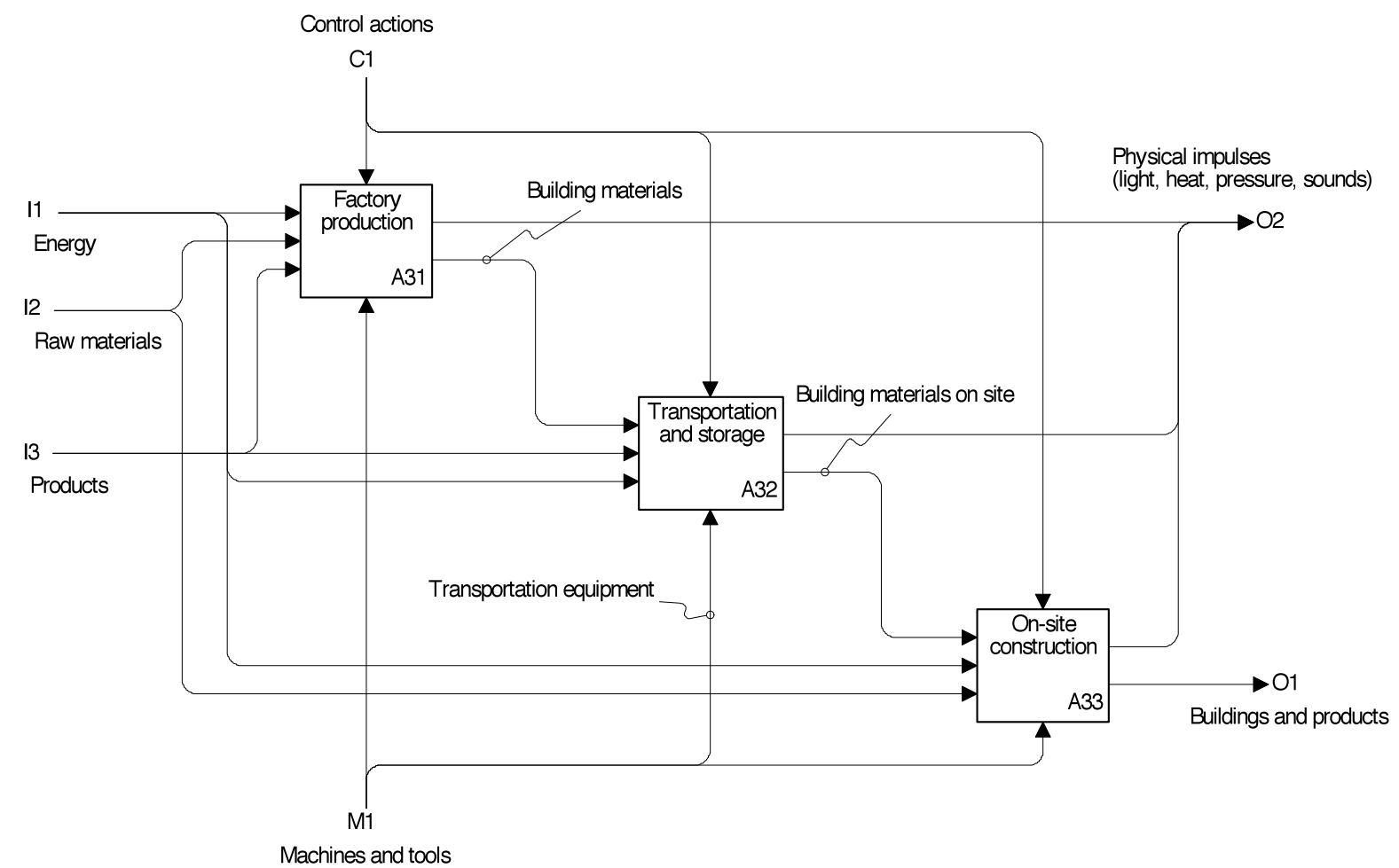

Figure 9. A subdivision of the material activities of the construction process

The author believes that the model presented above is quite generic in the sense that it models the overall construction process from a purely functional, technology neutral viewpoint. The only other author in the construction IT research literature who has discussed using the approach of a strict split between information and materials management is Tolman [1991].

\section{Acknowledgements}

The original impulse for this model came in 1994 through the need to develop a comprehensive IT in construction course for fourth year civil engineering students. After that the model has over the years slowly grown more complex and parts of it have been discussed with several colleagues, for instance during invited keynote presentations at conferences in Glasgow [Björk 1996] and Cairns [1997]. Some parts of the model have also been included as a background to an overall discussion of the scope and methods of construction information technology [Björk 1999].

\section{REFERENCES}

Björk, B.-C. 1996. Information Technology in Construction. Keynote lecture at: International conference on information technology in civil and structural engineering design - taking stock and future directions, organised by CIB W65, Glasgow, 14-16.8. 1996

Björk, B.-C. 1997 INFOMATE: A framework for discussing information technology applications in construction, keynote lecture, In: Drogemuller, R. edt. Information Technology Support for Construction Process Re-Engineering IT-CPR-97, CIB W78 Workshop, Cairns 9 - 11 July 1997.

Björk, B.-C. 1999, Information Technology in Construction - domain definition and research issues, International Journal for Computer Integrated Design and Construction, Vol 1, Issue 1, 1999, pp. 3-16 
Brandon, P. and Betts, M. 1995 (edts.) Integrated construction information. E\&FN Spon, London.

Goncalvec, R. and Steiger-Garcao, A. (edts.) 2000, Product and Process Modelling in Building and Construction, A. A. Balkema, Rotterdam,.

ICAM. 1981. Integrated computer-aided manufacturing (ICAM), Architecture Part II. Volume IV - Function modelling manual (IDEF0). SofTech., Waltham, MA, US.

ISO 1999; ISO/DIS 12006-2, Organisation of information about construction works - part 2: Framework for classification of information

Kaglioglou, M., Cooper, R., Aouad, G. and Sexton, M. 2000, Rethinking construction: the Generic Design and Construction Process Protocol, Engineering, Construction and Architectural Management, Vol 7/2, pp. 141-153

Karhu, V. and Lahdenperä, P. 1999, A formalised process model of current Finnish design and construction practice, International journal of construction information technology, Vol. 7 No 1, pp. 51-71.

Marca, D. A. and McGowan, C. L. 1987. SADT - structured analysis and design technique. New York, McGraw-Hill.

Merendonk, P. van \& Dissel, D. van. (eds.). 1989. Bouw informatie model; versie 3.1. IOPBouw. App., Rotterdam, Netherlands.

Sanvido, V., Khayyal, S., Guvenis, M., Norton, K., Hetrick, M., Al-Muallem, M., Chung, E., Medeiros, D., Kumara, S. \& Ham, I. 1990. An integrated building process model. Technical Report no 1, CIC Research Program. The Pennsylvania State University. University Park, PA, US.

Tolman, F. 1991: Some integration reqirements for computer integrated building. In: Preproceedings of the CIB W78 seminar The Computer Integrated Future, Eindhoven University of Technology, ,the Netherlands

Turk, Ziga 1997. Communication technologies in construction, Preproceedings or the Global Construction IT Futures Conference, The Construct IT Centre, University of Salford, UK, http://www.surveying.salford.ac.uk/meeting/docs/Abstarcts.htm

Webster's 1979. Webster's New Universal Unabridged Dictionary, 2nd edition, Simon and Schuster, New York. 